\title{
О КИПЕНИИ НА ПОВЕРХНОСТИ С ПОРИСТЫМ ПОКРЫТИЕМ
}

\author{
(Представлена И. Эпиком)
}

При кипении жидкости на поверхности, покрытой слоем лиофильного пористого материала толщиной $L$, жидкость двигается через пористый материал к поверхности нагрева под воздействием капиллярных сил, а пар - от поверхности нагрева под воздействием градиента давления. Увеличение тепловой нагрузки ведет к образованию новых паровых пор. При этом проницаемость пористого слоя для пара улучшается, а для жидкости, напрстив, ухудшается. Когда сила суммарного сопротивления пористого слоя течению жидкости и пара становится больше силы максимального капиллярного давления, жидкость перестает доходить до поверхности нагрева, и часть пористого материала осушается. Будем называть это состояние внутрислойным кризисом в отличие от кризиса кипения, при котором жидкость отделяется от поверхности нагрева слоем пара.

Если пренебречь гравитационными силами, ввиду их малости по сравнению с капиллярными силами, то условие внутрислойного кризиса выразится балансом капиллярных сил и сил сопротивления движению жидкости и пара в пористом материале:

$$
\frac{2 \sigma}{a_{\text {мин }}}=\frac{\mu_{L} \bar{w}_{L} L}{k_{L}}+\frac{\mu_{V} \bar{w}_{V} L}{k_{V}} \frac{p_{1}}{\bar{p}},
$$

где $a$ - радиус пор, $\mu-$ динамическая вязкость, $\sigma$ - поверхностное натяжение, $\bar{w}-$ приведенная скорость, $k-$ проницаемость, $p_{1}-$ давление в большом объеме, $\bar{p}$ - среднее давление пара в пористом материале, индексы $L$ и $V$ означают жидкость и пар соответственно. Первый член в уравнении (1) описывает максимальную величину капиллярной тяги, второй - сопротивление трению для жидкости и третий - coпротивление трению для пара по закону Дарси.

Заменим пористое покрытие его моделью с прямыми цилиндрическими капиллярами длиной, равной толщине пористого слоя, и с такой же пористостью и распределением пор по размерам, как у реального пористого материала. Капилляры радиусом $a \leqslant 2 \sigma / \Delta p$ заполнены жидкостью, остальные - паром. Стенки паровых капилляров покрыты микрослоем жидкости толщиной $\delta$.

В соответствии с законом Дарси и с учетом того, что весь тепловой поток идет на парообразование, перепад давлений в пористом слое можно записать в виде 


$$
\Delta p=\frac{q_{\mu_{V} L} L}{k_{V} r_{\mathrm{Q} V}} \frac{p_{1}}{\bar{p}}
$$

где $q$ - величина тепловой нагрузки, $\varrho v-$ плотность пара, $r-$ скрытая теплота парообразования.

Перепад между температурами на поверхности нагрева под пористым слоем и кипящей жидкости представим состоящим из трех компонентов (рис. 1): 1) разность температур между поверхностью нагрева и паром в пористом материале $\left.\Delta T_{1} ; 2\right)$ то же между паром в пористом материале и паром в большом объеме $\left.\Delta T_{2} ; 3\right)$ то же на участке сухого пористого слоя $\Delta T_{3}$.

Для расчета $\Delta T_{1}$ проанализируем тепловой режим стержня, длина которого равна толщине пористого покрытия. Периметр стержня определим исходя из удельной открытой поверхности пористого материала. Стержень тонкий, поэтому температуру по всему его поперечному сечению принимаем постоянной. Температура сечения зависит от того, на каком расстоянии от основания стержня оно находится. Разность тепловых потоков между двумя параллельными сечениями равняется количеству тепла, отданного в

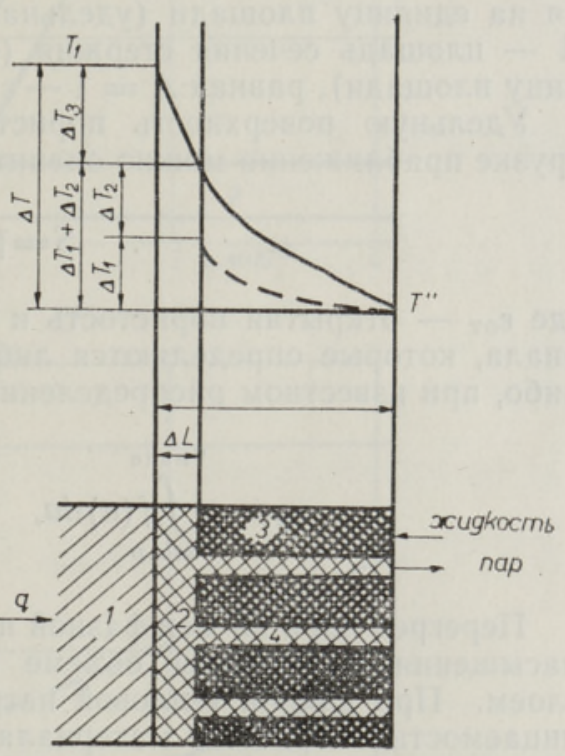

Рэс. 1. Распределение температуры в перистом слое: 1 - поверхность нагрева, 2 - сухая прослойка, 3 - жидкостные поры, 4 - паровые поры. окружающую среду с данного участка стержня.

Для рассматриваемых условий уравнение баланса имеет вид

$$
\frac{d}{d x}\left(\lambda A \frac{d T}{d x}\right)=\alpha\left(T-T_{0}\right) U .
$$

Согласно $\left[{ }^{2}\right]$, решение этого уравнения для температуры сечения, расположенного на расстоянии $x$ от основания призматического стержня, представим в виде

$$
T_{x}=T_{0}+T_{1}-T_{0}\left[\frac{(1-n) \mathrm{e}^{-m(L-x)}+(1+n) \mathrm{e}^{m(L-x)}}{2[\operatorname{ch}(m L)+n \operatorname{sh}(m L)]}\right] .
$$

Температура на свободном конце стержня (на внешней поверхности порнстого слоя) равняется

$$
T=T_{0}+\frac{T_{1}-T_{0}}{\operatorname{ch}(m L)+n \operatorname{sh}(m L)},
$$

а перепад между температурами на основании стержня $T_{1}$ и средой охлаждения $T_{0}$ (на поверхности нагрева п в паровых порах) -

$$
\Delta T_{1}=q / \lambda m A \frac{n+\operatorname{th}(m L)}{1-i-n \operatorname{th}(m L)},
$$


где $m$ - характеристика стержня (пористого слоя), равная $m=\sqrt{\alpha U / \lambda A}$; $n$ - характеристика торца стержня (внешней поверхности пористого слоя), равная $n=\alpha_{L} / \lambda m ; \alpha_{L}$ - коэффициент теплоотдачи с торца стержня; $\alpha$ - коэффициент теплоотдачи с боковой поверхности стержня, равный $\alpha=\lambda_{L} / \delta ; \lambda$ и $\lambda_{L}-$ коэффициенты теплопроводности сухого пористого материала и жидкости соответственно; $U$ - периметр стержня на единицу площади (удельная открытая внутренняя поверхность); $A$ - площадь сечения стержня (скелета пористого материала на единицу площади), равная $A=1-\varepsilon$, где $\varepsilon-$ пористость.

Удельную поверхность пористого слоя при данной тепловой нагрузке приближенно можно оценить по формуле Козени

$$
S=\sqrt{c \varepsilon_{\mathrm{OT}}^{3} / k_{V}}
$$

где $\varepsilon_{\text {от }}$ - открытая пористость и $k_{V}$ - проницаемость пористого материала, которые определяются либо экспериментально по методике [ $\left.{ }^{1}\right]$, либо, при известном распределении пор по размерам $f(a)$, по формулам

$$
\varepsilon_{\text {от }}=\varepsilon \int_{2 \sigma / \Delta p}^{a_{\mathrm{Mакс}}} f(a) d a, \quad k_{V}=\frac{\varepsilon}{8} \int_{2 \sigma / \Delta p}^{a_{\mathrm{Mа \pi с}}} a^{2} f(a) d a .
$$

Перегрев пара на межфазной поверхности относительно температуры насыщения в большом объеме зависит от давления под пористым слоем. При данной тепловой нагрузке это давление определяется проницаемостью пористого материала для пара и, в соответствии с законом Дарси, описывается выражением (2). Ради простоты мы не учитываем сжимаемость пара, так как перепад давлений при течении пара через пористое покрытие пренебрежимо мал. Тогда на основе закона Клаузиуса-Клапейрона из (2) получаем

$$
\Delta T_{2}=\frac{T^{\prime \prime} q \mu_{V} L}{k_{V} r^{2} Q_{V}^{2}}
$$

Когда жидкость перестает доходить до поверхности нагрева, образуется сухой пористый слой толщиной

$$
\Delta L=\frac{2 \sigma r}{a_{\text {мин }}\left(\frac{v_{L}}{k_{L}}+\frac{v_{V}}{k_{V}} \frac{p_{1}}{\bar{p}}\right)}\left(\frac{1}{q_{*}}-\frac{1}{q}\right),
$$

где $q_{*}$ - максимальная величина тепловой нагрузки в соответствии с равенством (1). нием

На участке сухого слоя перепад температур определяется выраже-

$$
\Delta T_{3}=q \Delta L / \lambda
$$

Таким образом, перепад между температурами на поверхности нагрева и кипящей жидкости в большом объеме будет равен

$$
\Delta T=\Delta T_{1}+\Delta T_{2}+\Delta T_{3} .
$$

В целях определения оптимальной толщины пористого материала и оценки влияния его теплопроводности на перепад температур между 

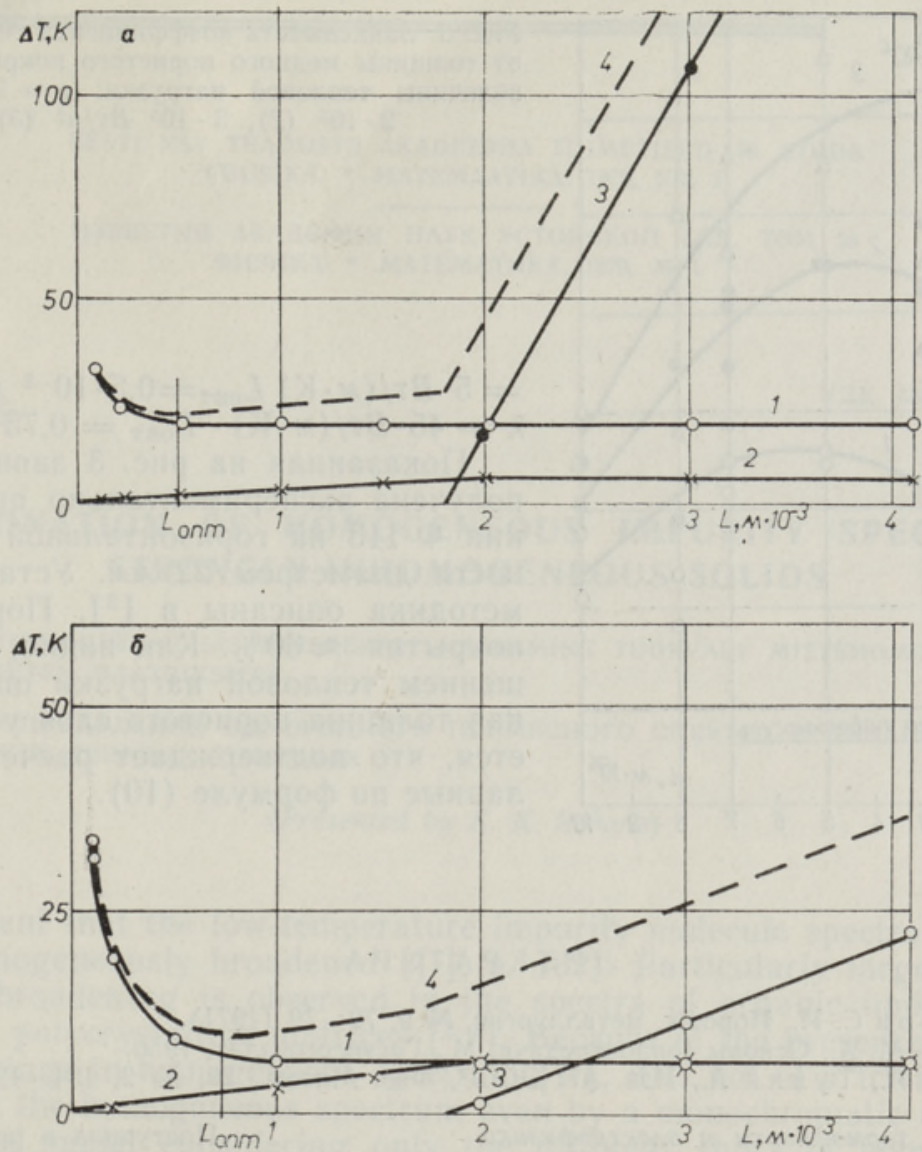

Рис. 2. Зависимость температурного напора между повсрхностью нагрева и жидкостью от толщины пористого слоя: $a$ - при $\lambda=5 B T /(\mu \cdot \mathrm{K}) ; \sigma-$ при $\lambda=45 B T /(\mu \cdot \mathrm{K}) .1-\Delta T_{1}, 2-\Delta T_{2}$, $3-\Delta T_{3}, 4-\Delta T$.

поверхностью нагрева и кипящей жидкостью были выполнены расчеты по формулам (4)-(10) для случая кипения трифтортрихлорэтана (Ф-113) при следующих условиях: $q=3,92 \cdot 10^{5} \mathrm{BT} / \mathrm{M}^{2} ; \varepsilon=0,3 ; \varepsilon_{o т}=$ $=0,073 ; k_{V}=0,276 \cdot 10^{-12} \mu^{2}$ при указанной тепловой нагрузке; $\delta=$ $=5 \cdot 10^{-6} \mu ; \lambda=5$ и $45 \mathrm{BT} /(\mu \cdot \mathrm{K}) ; \alpha_{L}=5000 \mathrm{BT} /\left(\mu^{2} \cdot \mathrm{K}\right)$.

Результаты расчета показали (см. рис. 2 и 3), что составляющая $\Delta T_{1}$ уменьшается с увеличением толщины покрытия, $\Delta T_{2}$ зависит линейно от толщины покрытия, если считать, что толщина активного слоя, где происходит испарение, значительно меньше толщины всего слоя. Начиная с определенной толщины $\Delta T_{2}$ перестает изменяться, так как радиус кривизны менисков сокращается до минимума. Выбранная нагрузка $q=3,92 \cdot 10^{5} B T / \mu^{2}$ является максимальной для внутрислойного кризиса при толщине пористого слоя $L *=1,8 \cdot 10^{-3}$ м. В случае бо́льшей толщины появляется и составляющая $\Delta T_{3}$. Результирующая кривая $\Delta T=f(L)$ имеет минимум, абсцисса которого определяет оптимальную толщину пористого покрытия при данной тепловой нагрузке, а, следовательно, и оптимальные условия теплоотдачи. При одинаковых структурных характеристиках оптимальная толщина больше у пористого материала с высокой теплопроводностью, Так, при $\lambda=$ 


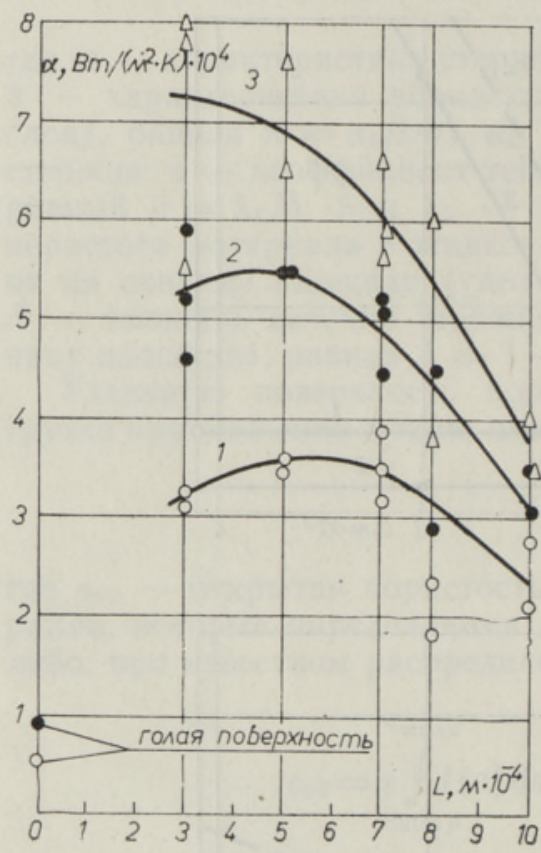

Рис. 3. Зависимость коэффициента теплоотдачи от толщины медного пористого покрытия и от величины тепловой нагрузки: $q=1 \cdot 10^{5}(I)$, $2 \cdot 10^{5}(2), 3 \cdot 10^{5} B T / M^{2} \quad(3)$.

$=5 B T /(\mu \cdot \mathrm{K}) L_{\text {опт }}=0,5 \cdot 10^{-3} \mu$, а при $\lambda=45 B T /(\mu \cdot \mathrm{K}) \quad L_{\text {опт }}=0,75 \cdot 10^{-3} \mu$. Показанная на рис. 3 зависимость получена экспериментально при кипении Ф-113 на горизонтальной поверхности диаметром 22 мм. Установка и методика описаны в [ $\left.{ }^{3}\right]$. Пористость покрытия $\approx 60 \%$. Как видно, с повышением тепловой нагрузки оптимальная толщина пористого слоя уменьшается, что подтверждает расчеты, сделанные по формуле (10).

\section{Л И ТЕРА Т У Р А}

1. В и к т о р о в С. И., Порошк. металлургия, № 9, $72-79$ (1971).

2. М и хе е в М. А., Основы теплопередачи, М., Госэнергоиздат, 1956.

3. Т е х вер Я., Т уник А., Изв. АН ЭССР, Физ. Матем., 26, № 2, $194-198$ (1977).

Ннститут термофизики и электрофизики Академии наук Эстонской ССР
Поступила в редакцио $11 /$ XI 1977

\section{J. TEHVER, A. TUNIK}

\section{KEEMINE POORSE KATTEGA PINNAL}

Artiklis on analüüsitud poorse pinnakatte parameetrite mõju soojusülekandele dielektriliste vedelike keemisel loomuliku konvektsiooni korral. On leitud pinna ülekuumenduse sõltuvus pinnakatte paksusest, poorsusest ning soojusjuhtivusest.

\section{J. TEHVER, A. TUNIK}

\section{CONCERNING BOILING ON POROUS COATING}

In the present paper the influence of parameters of a porous surface coating on heat transfer for boiling dielectric fluids is analyzed. The formulae that characterize the dependence of heat transfer intensity on thickness of surface coating, porosity and heat conductivity are obtained by a simplified model for porous material and process of boiling. The obtained relations enable us to show the existence of the optimum thickness of porous layer at which the heat transfer has the highest intensity. The optimum thickness of the layer decreases with the fall of heat conductivity and the increase of heat load. Experimental data on heat transfer for the boiling of F-113 on surfaces with porous copper coating confirming the theoretically obtained relationships, are given in the work as well. 